\title{
Do nicotine dependence influencing and non-influencing behaviors have an association with high nicotine dependence in smokers?
}

\author{
Zhongbo Chen ${ }^{1+*}$, Xuechan Yu ${ }^{1+*}$, Dan $\mathrm{Lv}^{1}$, Liang Zhang ${ }^{2}$, Hanlu Gao ${ }^{3}$, Younuo Wang ${ }^{1}$, Qingwen Su${ }^{3}$, Hongying Ma ${ }^{1}$, Jie \\ Chen', Hui Chen', Qidong Zhuang ${ }^{1}$, Panfeng Fu', Yiming Yu', Zaichun Deng ${ }^{1}$
}

\begin{abstract}
INTRODUCTION Nicotine dependence (ND) is a maladaptive pattern of tobacco smoking with withdrawal symptoms similar to other drug addictive disorders. It is very common in clinical practice that smokers always have different degrees of nicotine dependence with the same amount of tobacco consumption. Behaviors may influence daily cigarette consumption or smoking status. Hence it is critical to ascertain the association between concurrent behaviors and high nicotine dependence among smokers.

METHODS A total of 343 patients who attended a clinic for smoking cessation were recruited, and the information on concurrent behaviors were recorded. Factors associated and not associated with nicotine dependence were recorded. Nicotine dependence was determined by Fagerström test for nicotine dependence (FTND). RESULTS High ND patients (FTND >5) showed significant behaviors distribution compared with mild and moderate ND patients (FTND $\leq 5$ ). There is no single behavior that was significantly different between high ND and mild and moderate ND smokers. However, the combined effects of nicotine dependence influencing behaviors of caffeine drinking and mental activities after dinner have an association with high ND $(\mathrm{OR}=1.939 ; 95 \%$ CI: $1.154-3.258, \mathrm{p}=0.012)$. In addition, the combined effects of inadequate sleep time ( $<8$ hours), caffeine drinking and mental activities after dinner significantly distinguished patients of high ND from those of low ND (OR=2.208; 95\% CI: 1.032-4.737, $\mathrm{p}=0.042)$.

CONCLUSIONS Interaction effects of mental activities after dinner and caffeine drinking have an association with high nicotine dependence. Sleep of less than 8 hours with behaviors of mental activities after dinner and caffeine drinking have the same effect.
\end{abstract}

AFFILIATION

1 Department of Pulmonary and Critical Care Medicine, The Affiliated Hospital of Medical School of Ningbo University, Ningbo, China 2 Ningbo Municipal Center for Disease Control and Prevention, Ningbo, China 3 Department of Prevention and Health Care, The

Affiliated Hospital of Medica School of Ningbo University,

Ningbo, China

+Co-first authors

${ }^{*}$ Contributed equally

CORRESPONDENCE TO

Yiming Yu. Department of Pulmonary and Critical Care Medicine, The Affiliated Hospital of Medical School of Ningbo University, No. 247, Renmin Road, Jiangbei District, Ningbo, Zhejiang Province, China. E-mail:yuyiming0205@163.com ORCID ID: http://orcid. org/0000-0003-3035-5649

Zaichun Deng. Department of Pulmonary and Critical Care Medicine, The Affiliated Hospital of Medical School of Ningbo University, No. 247, Renmin Road, Jiangbei District, Ningbo, Zhejiang Province, China.

E-mail: dengzaichun@nbu. edu.cn

KEYWORDS

behaviors, sleep, high nicotine dependence, caffeine drink, mental activities after dinner

Received: 29 May 2021

Revised: 27 September 2021

Accepted: 4 October 2021 


\section{INTRODUCTION}

Tobacco smoking is a preventable human behavior and has been identified to play a critical role in the pathogenesis of various malignant cancers ${ }^{1}$ and pulmonary diseases such as chronic obstructive pulmonary disease (COPD $)^{2}$. Nicotine dependence (ND) is a maladaptive pattern of tobacco smoking with withdrawal symptoms similar to other drug addictive disorders; and has been defined by the Diagnosis and Statistical Manual of Mental Disorders-edition IV (DSM-IV) ${ }^{3}$. In China, the smoking rate of men (53.3\%, 95\% CI: $51.4-55.2)$ is much higher than that of women $(2.5 \%, 95 \%$ CI: $1.9-3.0)(\mathrm{p}<0.05)^{4}$. Moreover, the proportion of smoking quitters in China is low, and the awareness of quitting is weak ${ }^{4}$. Previous studies have shown that different behaviors are closely related to smoking status change and cigarette consumption ${ }^{5}$. Caffeine withdrawal makes it more difficult to quit smoking ${ }^{6}$. Alcohol and nicotine dependence are likely to co-occur, resulting in a lower likelihood of smoking cessation ${ }^{7,8}$. People with behaviors that do not influence nicotine dependence, including exercise and enough sleep, may consume fewer cigarettes daily and have a lower nicotine dependence $^{9-12}$. Influencing behaviors are those that can increase smoking or increase the level of nicotine dependence. Non-influencing behaviors are those that have no influence on smoking cigarettes or nicotine dependence. Moreover, it is very common in clinical practice that smokers have different degrees of nicotine dependence with the same tobacco consumption. Therefore, we investigated, by recording smokers' behaviors as medical history, whether there is a relationship between influencing and noninfluencing behaviors of nicotine dependence and high nicotine dependence. However, so far there is no evidence showing that influencing behaviors increase the risk of high ND or vice versa.

\section{METHODS}

Information on the lifestyle factors of 343 smokers who came to our clinic to quit smoke were recorded from April 2010 to January 2020 in the Affiliated Hospital of Medical School of Ningbo University. All research subjects provided informed consent, and the research study was approved by the Ethics Committee of the Affiliated Hospital. All methods were carried out following NCCN Clinical Practice Guidelines in
Oncology of Smoking Cessation ${ }^{13}$.

\section{Survey design}

Clinical characteristics and lifestyle behaviors

Demographic (age, gender, BMI) data were recorded as clinical characteristics. We recorded the lifestyle behaviors of smokers before providing personalized treatment plans to patients in the smoking cessation clinic. We selected smokers' lifestyle behaviors, as identified in previous studies, that could influence cigarette consumption and nicotine dependence level. Moreover, these behaviors are easy to adjust or change during the progress of smoking cessation. Behaviors that have no effect on nicotine dependence were recorded as follows: exercise more than three times a week and each time more than 30 minutes $^{9,10}$; sleep time $\geq 8$ hours; taking an afternoon nap; and any type of fruit consumption $\geq 3$ times/week. In contrast, behaviors that can have an influence on nicotine dependence were regarded as the following: sleep time $<8$ hours; alcohol consumption more than $50 \mathrm{~mL} \geq 3$ times/week (40\% alcohol), caffeine drink intake $>200 \mathrm{~mL}$ every day; and mental activities after dinner before sleep ${ }^{14}$ (e.g. gambling, overtime work, video games, studying or other mental activities). All information was recorded on our self-designed form (Supplementary file).

\section{Measurement of nicotine dependence}

Nicotine dependence was measured by the Fagerström test for nicotine dependence (FTND) ${ }^{15,16}$. The FTND score was classified across five levels of dependence: 0-2 very low, 3-4 low , 5 moderate, 6-7 high, and $8-10$ very high ${ }^{17}$. We dichotomized the level of dependence into high (FTND >5) and low (FTND $\leq 5)$.

\section{Statistical analysis}

The behaviors information and results for 343 patients were included in the statistics. The t-test and mean \pm standard deviation (SD) were used in comparisons of age and BMI $\left(\mathrm{kg} / \mathrm{m}^{2}\right)$. Gender distribution and lifestyle behaviors across the two groups (FTND $\leq 5$ and FTND $>5$ ) were calculated by chi-squared analysis. Binary logistic analysis was used to estimate all independent or interaction effects of behaviors. The analysis was conducted using IBM SPSS Statistics 21.0, and p $<0.05$ was considered as statistically significant. 


\section{RESULTS}

Demographics of the study population are shown in Table 1. There were no differences noted in age, gender and BMI between the high and low nicotine dependence smokers. Influencing and non-influencing behaviors were differently distributed in the FTND $>5$ group (Table 2) $(\mathrm{p}=0.002)$, indicating that exercise, sleep, sleep time $\geq 8 \mathrm{~h}$ and fruit consumption were not associated with high nicotine dependence, neither independently nor combined (Table 3 ). Results in Table 4 show that caffeine drinking combined with mental activities after dinner were associated with
Table 1. Clinical characteristics of low (FTND $\leq 5)$ and high (FTND >5) nicotine dependence smokers, China $(\mathrm{N}=343)$

\begin{tabular}{l|c|c|c|}
\hline $\begin{array}{l}\text { Characteristics } \\
\text { Age (years) }\end{array}$ & $\begin{array}{c}\text { FTND } \leq 5>5 \\
(n=151)\end{array}$ & $p$ \\
$\begin{array}{l}\text { Mean } \pm \text { SD } \\
\text { Gender }(n)\end{array}$ & $42.6 \pm 11.0$ & $43.1 \pm 11.1$ & 0.664 \\
\hline $\begin{array}{l}\text { Male } \\
\text { Female }\end{array}$ & 141 & 176 & 0.682 \\
BMI $\left(\mathrm{kg} / \mathrm{m}^{2}\right)$ & 10 & 16 & \\
Mean \pm SD & $23.5 \pm 2.7$ & $23.6 \pm 7.7$ & 0.796
\end{tabular}

Table 2. Co-existence of both nicotine dependence influencing and non-influencing behaviors among low and high dependence smokers

Number of combined nicotine influencing behaviors and number of smokers with FTND $\leq 5$

Number of combined nicotine dependence non-

influencing behaviors among

FTND $\leq 5$ smokers

$\begin{array}{rrrrrrr} & 0 & 1 & 2 & 3 & 4 & 0.139 \\ 0 & 0 & 4 & 14 & 3 & 0 & \\ 1 & 11 & 18 & 24 & 7 & 1 & \\ 2 & 7 & 9 & 20 & 8 & 2 & \\ 3 & 3 & 11 & 4 & 1 & 0 & \\ 4 & 0 & 2 & 2 & 0 & 0 & \end{array}$

Number of combined nicotine dependence influencing behaviors and number of smokers with FTND $>5$

Number of combined nicotine dependence non-

influencing behaviors among FTND $>5$ smokers

$\begin{array}{rrrrcrl} & 0 & 1 & 2 & 3 & 4 & 0.002^{*} \\ 0 & 0 & 7 & 13 & 14 & 3 & \\ 1 & 8 & 30 & 27 & 7 & 3 & \\ 2 & 8 & 19 & 20 & 7 & 0 & \\ 3 & 6 & 7 & 7 & 1 & 0 & \\ 4 & 1 & 3 & 1 & 0 & 0 & \end{array}$

${ }^{*} \mathrm{p}<0.05$ was considered as statistically significant.

Table 3. The association between nicotine dependence non-influencing behaviors and high (FTND >5) nicotine dependence

\begin{tabular}{|c|c|c|}
\hline Behaviors & OR $(95 \% \mathrm{CI})$ & $p$ \\
\hline Exercise more than three times a week and each time more than 30 minutes & $0.820(0.521-1.291)$ & 0.392 \\
\hline Sleep time $\geq 8 \mathrm{~h}$ & $0.943(0.641-1.447)$ & 0.787 \\
\hline Afternoon nap & $1.081(0.684-1.708)$ & 0.74 \\
\hline Fruit consumption $\geq 3$ times/week & $0.683(0.415-1.124)$ & 0.133 \\
\hline \multicolumn{3}{|l|}{ Combination of two behaviors } \\
\hline Exercise + sleep time $\geq 8 \mathrm{~h}$ & $0.779(0.444-1.336)$ & 0.384 \\
\hline Exercise + afternoon nap & $1.072(0.596-1.928)$ & 0.818 \\
\hline Exercise + fruits & $0.576(0.298-1.111)$ & 0.1 \\
\hline Sleep time $\geq 8 \mathrm{~h}+$ afternoon nap & $1.118(0.603-2.075)$ & 0.723 \\
\hline Sleep time $\geq 8 \mathrm{~h}+$ fruits & $0.927(0.497-1.729)$ & 0.811 \\
\hline Afternoon nap + fruits & $1.079(0.480-2.422)$ & 0.855 \\
\hline \multicolumn{3}{|l|}{ Combination of three behaviors } \\
\hline Exercise + sleep time $\geq 8 \mathrm{~h}+$ fruits & $0.772(0.337-1.771)$ & 0.542 \\
\hline Exercise + sleep time $\geq 8 \mathrm{~h}+$ afternoon nap & $0.924(0.402-2.126)$ & 0.853 \\
\hline Exercise + fruits + afternoon nap & $1.012(0.368-2.782)$ & 0.982 \\
\hline Afternoon nap + fruits + sleep time $\geq 8 \mathrm{~h}$ & $1.105(0.344-3.553)$ & 0.867 \\
\hline \multicolumn{3}{|l|}{ Combination of four behaviors } \\
\hline Exercise + sleep time $\geq 8 \mathrm{~h}+$ fruits + afternoon nap & $0.983(0.259-3.724)$ & 0.979 \\
\hline
\end{tabular}


Table 4. The association between nicotine dependence influencing behaviors and high (FTND >5) nicotine dependence

\begin{tabular}{|c|c|c|}
\hline Behaviors & OR $(95 \%$ CI $)$ & $p$ \\
\hline Sleep time $<8 \mathrm{~h}$ & $1.061(0.691-1.628)$ & 0.787 \\
\hline Alcohol consumption more than $50 \mathrm{~mL} \geq 3$ times/week & $0.748(0.425-1.318)$ & 0.315 \\
\hline Caffeine drink intake $>200 \mathrm{~mL}$ every day & $0.869(0.566-1.336)$ & 0.523 \\
\hline $\begin{array}{l}\text { Mental activities after dinner (e.g. gambling, overtime work, video games, studying } \\
\text { or other mental activities) }\end{array}$ & $1.475(0.956-2.275)$ & 0.079 \\
\hline Sleep time $<8 \mathrm{~h}+$ alcohol consumption & $1.236(0.561-2.725)$ & 0.599 \\
\hline Sleep time $<8 \mathrm{~h}+$ caffeine drink & $1.011(0.622-1.645)$ & 0.964 \\
\hline Sleep time $<8 \mathrm{~h}+$ mental activities after dinner & $1.142(0.671-1.945)$ & 0.624 \\
\hline Alcohol consumption + caffeine drink & $0.675(0.338-1.349)$ & 0.266 \\
\hline Alcohol consumption + mental activities after dinner & $0.481(0.202-1.144)$ & 0.098 \\
\hline Caffeine drink + mental activities after dinner & $1.939(1.154-3.258)$ & $0.012^{*}$ \\
\hline Sleep time $<8 \mathrm{~h}+$ alcohol consumption + caffeine drink & $1.494(0.581-3.843)$ & 0.405 \\
\hline Sleep time $<8 \mathrm{~h}+$ alcohol consumption + mental activities after dinner & $0.914(0.301-2.780)$ & 0.875 \\
\hline Sleep time $<8 \mathrm{~h}+$ caffeine drink + mental activities after dinner & $2.208(1.032-4.737)$ & $0.042^{*}$ \\
\hline Alcohol consumption + caffeine drink + mental activities after dinner & $0.676(0.240-1.909)$ & 0.46 \\
\hline $\begin{array}{l}\text { Sleep time }<8 \mathrm{~h}+\text { alcohol consumption + caffeine drink + mental activities after } \\
\text { dinner }\end{array}$ & $1.591(0.391-6.470)$ & 0.516 \\
\hline
\end{tabular}

${ }^{*} p<0.05$ was considered as statistically significant.

high nicotine dependence $(\mathrm{OR}=1.939 ; 95 \% \mathrm{CI}$ : $1.154-3.258, \mathrm{p}=0.012$ ). Three unhealthy interactionbehaviors ( sleep time $<8$ hours, caffeine drinking and mental activities after dinner) were associated with high nicotine dependence $(\mathrm{OR}=2.208 ; 95 \%$ CI: $1.032-4.737, \mathrm{p}=0.042$.

\section{DISCUSSION}

Nicotine dependence is a maladaptive pattern of tobacco smoking with withdrawal symptoms like other drug addictive disorders. Many behaviors may change the daily consumption of cigarettes or smoking status5. People with non-influencing behaviors such as exercising and adequate sleep have lower nicotine dependence $^{9,18}$. Previous studies have demonstrated that influencing behaviors such as insufficient sleep duration $^{11,18}$, caffeine drink consumption ${ }^{6,19}$, alcohol dependence $^{7,8}$, and mental activities (e.g. gambling ${ }^{20,21}$, video games ${ }^{22}$, overtime working ${ }^{23}$ ) increase cigarette consumption and can change ex-smokers or nonsmokers into smokers. However, one needs to take into account the smokers' lifestyle. There are few studies that have investigated the influence of combined lifestyle behaviors on high nicotine dependence. To our knowledge, our research is first to reveal the association between nicotine dependence influencing and non-influencing behaviors and high ND. Importantly, we showed that the interaction of mental activities after dinner, caffeine drinking and sleep time $<8$ hours are associated with high ND.

During the night without smoking, a decrease in nicotine level can recover nicotinic-acetylcholine receptors. It may contribute to the development of withdrawal symptoms in the morning when craving for cigarettes ${ }^{24}$. Further, inadequate sleep duration decreases positive mood and increases negative $\operatorname{mood} 11$ and the dopamine caused by the calming effect of nicotine relieves this bad $\operatorname{mood}^{25}$. Therefore, high nicotine dependence smokers view the first few cigarettes of the day as the most pleasant and are thus not willing to quit smoking. We can also understand why sleep duration is associated with high ND.

Mental activities after dinner ${ }^{14}$ (e.g. gambling, overtime work, video games, studying or other mental activities) and smoking may be related to environment factors such as colleagues, classmates, gambling and video game playing patterns. Moreover, tobacco is used so that dopamine can alleviate anxiety and allow one to cope with stress in these situations ${ }^{26-28}$. Furthermore, coffee or caffeine intake is also a choice for dealing with tiredness by low-cost addictive products that are available anywhere and anytime. 
The prevalence of drinking caffeine beverages and smoking happen during mental activities after dinner or in the morning without adequate sleep time. Caffeine withdrawal symptoms increase the difficulty of smoking cessation ${ }^{19}$. So, the synergistic effect of mental activities after dinner, caffeine drinking and inadequate sleep duration increases the risk of high nicotine dependence.

Our data show that nicotine dependence noninfluencing habits are not associated with high ND. This result is different from previous studies that showed that smokers who exercise have lower nicotine dependence and lower tobacco consumption ${ }^{9,10}$. The main reason for the difference is that we chose smokers of all ages who exercise more than 3 times a week and each time more than 30 minutes. This inclusion criterion can be used for all high nicotine dependence smokers. We also showed that afternoon napping was not associated with high nicotine dependence; there are few studies showing the relationship between napping and nicotine dependence. Therefore, we suggest that smokers who come to the clinic for cessation help with high nicotine dependence should fully consider an individualized treatment, no matter their nicotine dependence influencing and non-influencing behaviors, instead of avoiding only influencing behaviors or developing non-influencing behaviors only.

We did not investigate education and occupation situation in this study, for two reasons. Firstly, in many studies, education and occupation were not found to be associated with higher cigarette consumption and higher nicotine dependence level. Secondly, we did not investigate these two factors because smokers need more feasible plans to assist in smoking cessation and improve the success rate of quitting smoking. Compared with change in lifestyle behaviors, it is more difficult to improve education or change jobs in order to quit smoking. Moreover, there are few studies about improving education or finding new jobs in order to change smoking status.

\section{Limitations}

We have one limitation in our research: the cigarette daily consumption and FTND should be investigated or studied in the future. This would help to better ascertain whether lifestyle behaviors can influence both cigarette consumption and nicotine dependence level. Furthermore, in China, the smoking rates of men are higher than the rates of women ${ }^{4}$ and awareness about quitting smoking is generally weak ${ }^{4}$. Therefore, information records on female smokers are few.

\section{CONCLUSIONS}

The interactive effects of mental activities after dinner and caffeine drinking were found to be associated with high nicotine dependence. Sleeping less than 8 hours combined with mental activities after dinner and caffeine drinking also indicated a similar association. Avoiding these behaviors may decrease the likelihood for high nicotine addiction and may help increase the possibility for smoking cessation. Smokers should decrease mental activities and caffeine drinking at night, which may contribute to a decrease in nicotine dependence. Individualized treatment for smokers should fully consider both healthy and unhealthy lifestyles.

\section{REFERENCES}

1. Siegel RL, Miller KD, Jemal A. Cancer Statistics, 2019. CA Cancer J Clin. 2019;69:7-34. doi:10.3322/caac.21654.

2. Willemse BW, Postma DS, Timens W, ten Hacken NH. The impact of smoking cessation on respiratory symptoms, lung function, airway hyperresponsiveness and inflammation. Eur Respir J. 2004;23(3):464-476. doi:10.1183/09031936.04.00012704

3. Robins LN, Helzer JE, Croughan J, Ratcliff KS. National Institute of Mental Health Diagnostic Interview Schedule. Its history, characteristics, and validity. Arch Gen Psychiatry. 1981;38(4):381-389. doi:10.1001/archpsyc.1981.01780290015001

4. Zhang M, Wang LM, Li YC, et al. Cross-sectional survey on smoking and smoking cessation behaviors among Chinese adults in 2010. Article in Chinese. Zhonghua Yu Fang Yi Xue Za Zhi. 2012;46(5):404-408. Accessed May 29, 2021. http://www.yiigle.com/LinkIn.do?linkin_ type $=$ pubmed $\&$ issn $=0253-9624 \&$ year $=2012 \&$ vol $=46 \&$ is sue $=5 \&$ fpage $=404$

5. Strine TW, Okoro CA, Chapman DP, et al. Healthrelated quality of life and health risk behaviors among smokers. Am J Prev Med. 2005;28(2):182-187. doi:10.1016/j.amepre.2004.10.002

6. Swanson JA, Lee JW, Hopp JW. Caffeine and nicotine: a review of their joint use and possible interactive effects in tobacco withdrawal. Addict Behav. 1994;19(3):229-256. doi:10.1016/0306-4603(94)90027-2

7. Falk DE, Yi HY, Hiller-Sturmhöfel S. An epidemiologic analysis of co-occurring alcohol and tobacco use and disorders: findings from the National Epidemiologic Survey on Alcohol and Related Conditions. Alcohol Res Health. 2006;29(3):162-171. Accessed May 29, 2021. https://www.ncbi.nlm.nih.gov/pmc/articles/ 


\section{PMC6527037/pdf/162-171.pdf}

8. Breslau N, Peterson E, Schultz L, Andreski P, Chilcoat H. Are smokers with alcohol disorders less likely to quit?. Am J Public Health. 1996;86(7):985-990. doi:10.2105/ajph.86.7.985

9. Audrain-McGovern J, Rodriguez D, Moss HB. Smoking progression and physical activity. Cancer Epidemiol Biomarkers Prev. 2003;12(11 Pt 1):1121-1129. Accessed May 29, 2021. https://cebp.aacrjournals.org/ content/12/11/1121.full-text.pdf

10. Loprinzi PD, Walker JF. Nicotine Dependence, Physical Activity, and Sedentary Behavior among Adult Smokers. N Am J Med Sci. 2015;7(3):94-99. doi:10.4103/1947-2714.153920

11. Hamidovic A, de Wit H. Sleep deprivation increases cigarette smoking. Pharmacol Biochem Behav. 2009;93(3):263-269. doi:10.1016/j.pbb.2008.12.005

12. Fucito LM, Redeker NS, Ball SA, Toll BA, Ikomi JT, Carroll KM. Integrating a Behavioural Sleep Intervention into Smoking Cessation Treatment for Smokers with Insomnia: A Randomised Pilot Study. J Smok Cessat. 2014;9(1):31-38. doi:10.1017/jsc.2013.19

13. Shields P, Herbst R, Arenberg D, et al. Smoking Cessation, Version 1.2016, NCCN Clinical Practice Guidelines in Oncology. J Natl Compr Canc Netw. 2016;14(11):14301468. doi:10.6004/jnccn.2016.0152

14. Yu X, Yu Y, Ma H, Chen Z, Deng Z. Mental activities after dinner increase cigarettes consumption. Sci Rep. 2021;11(1):2405. doi:10.1038/s41598-021-81978-y

15. Payne TJ, Smith PO, McCracken LM, McSherry WC, Antony MM. Assessing nicotine dependence: a comparison of the Fagerström Tolerance Questionnaire (FTQ) with the Fagerström Test for Nicotine Dependence (FTND) in a clinical sample. Addict Behav. 1994;19(3):307-317. doi:10.1016/0306-4603(94)90032-9

16. Ray R, Schnoll RA, Lerman C. Nicotine dependence: biology, behavior, and treatment. Annu Rev Med. 2009;60:247-260. doi:10.1146/annurev.med.60.041707.160511

17. Cao F, Cai L, Gui WL, Sun CH, He JQ. Socioeconomic difference in prevalence of nicotine dependence among Naxi ethnic minority in Yulong County of Yunnan Province. Article in Chinese. Chinese Journal of Disease Control and Prevention. 2016;20(05):452-255. doi:10.16462/j.cnki.zhjbkz.2016.05.006

18. Cohrs S, Rodenbeck A, Riemann D, et al. Impaired sleep quality and sleep duration in smokers-results from the German Multicenter Study on Nicotine Dependence. Addict Biol. 2014;19(3):486-496. doi:10.1111/j.1369-1600.2012.00487.x

19. Tanda G, Goldberg SR. Alteration of the behavioral effects of nicotine by chronic caffeine exposure. Pharmacol Biochem Behav. 2000;66(1):47-64. doi:10.1016/s0091-3057(00)00234-3

20. Grant JE, Potenza MN. Tobacco use and pathological gambling. Ann Clin Psychiatry. 2005;17(4):237-241.

\section{doi:10.1080/10401230500295370}

21. McGrath DS, Barrett SP. The comorbidity of tobacco smoking and gambling: a review of the literature. Drug Alcohol Rev. 2009;28(6):676-681. doi:10.1111/j.1465-3362.2009.00097.x

22. Raiff BR, Jarvis BP, Rapoza D. Prevalence of video game use, cigarette smoking, and acceptability of a video gamebased smoking cessation intervention among online adults. Nicotine Tob Res. 2012;14(12):1453-1457. doi:10.1093/ntr/nts079

23. Angrave D, Charlwood A, Wooden M. Working time and cigarette smoking: evidence from Australia and the United Kingdom. Soc Sci Med. 2014;112:72-79. doi:10.1016/j.socscimed.2014.04.031

24. Dani JA, Heinemann S. Molecular and cellular aspects of nicotine abuse. Neuron. 1996;16(5):905-908. doi:10.1016/s0896-6273(00)80112-9

25. Gilbert DG. Paradoxical tranquilizing and emotionreducing effects of nicotine. Psychol Bull. 1979;86(4):643661. doi:10.1037/0033-2909.86.4.643

26. Gray JA, Young AM, Joseph MH. Dopamine's role. Science. 1997;278:1548-1549. doi:10.1126/science.278.5343.1547b

27. DeWitt EE. Neuroeconomics: a formal test of dopamine's role in reinforcement learning. Curr Biol. 2014;24(8):R321-R324. doi:10.1016/j.cub.2014.02.055

28. Grant JE, Desai RA, Potenza MN. Relationship of nicotine dependence, subsyndromal and pathological gambling, and other psychiatric disorders: data from the National Epidemiologic Survey on Alcohol and Related Conditions. J Clin Psychiatry. 2009;70(3):334-343. doi:10.4088/jcp.08m04211

\section{CONFLICTS OF INTEREST}

The authors have completed and submitted the ICMJE Form for Disclosure of Potential Conflicts of Interest and none was reported.

\section{FUNDING}

Funding for this study was provided by The Affiliated Hospital of Medical School of Ningbo University Youth Talent Cultivation Program (Grant number: FYOM-LC-202003), Ningbo Social and Scientific Development Fund (Grant number: 2015C50012), Ningbo Health Youth Technical Key Talents Training Special Project2020SWSONGG-05and the Natural Science Foundation of Ningbo (Grant numbers: 2018A610271 and 2017A610250).

\section{ETHICAL APPROVAL AND INFORMED CONSENT}

All research subjects provided informed consent, and the research study was approved by the Ethics Committee of the Affiliated Hospital of Medical school of Ningbo University (Ningbo, China) with approval numbers NBU-2020-080, February 2020, and KY20210102, January 2021. All methods were carried out following NCCN Clinical Practice Guidelines in Oncology of Smoking Cessation.

\section{DATA AVAILABILITY}

The data supporting this research are available from the authors on reasonable request.

\section{PROVENANCE AND PEER REVIEW}

Not commissioned; externally peer reviewed. 